\title{
Fano and Kondo resonance in electronic current through nanodevices
}

\author{
Bogdan R. Bułka and Piotr Stefański \\ Institute of Molecular Physics, Polish Academy of Sciences, ul.Smoluchowskiego 17, 60-179 Poznań, Poland
}

(October 30, 2018)

\begin{abstract}
Electronic transport through a quantum dot strongly coupled to electrodes is studied within a model with two conduction channels. It is shown that multiple scattering and interference of transmitted waves through both channels lead to Fano resonance associated with Kondo resonance. Interference effects are also pronouncedly seen in transport through the Aharonov-Bohm ring with the Kondo dot, where the current characteristics continuously evolve with the magnetic flux.
\end{abstract}

PACS 73.63.-b, 72.15.Qm, 75.20.Hr

Recent electron transport experiments performed in a single electron transistor strongly coupled to electrodes [1] and by a scanning tunneling microscope (STM) on a single magnetic adatom on a metallic surface [2,3] showed that the Kondo resonance [4] occurs simultaneously with the Fano resonance [5]. Multiple scatterings of travelling electronic waves on a localized magnetic state are crucial for a formation of both resonances. The condition for the Fano resonance to appear is a presence of at least two scattering channels: the discrete level and the broad continuum band [5,6]. In the mesoscopic systems the nature of two conduction channels is dependent on the geometry of the device under consideration. Interferometer geometry is realized when an Aharonov-Bohm ring with a quantum dot (QD) placed in one of the arms is studied [7]. When an adatom is deposited on the metallic surface, the STM tip probes indirectly the hybridized local adatom level together with the band of surface electrons [2, 3, 10,11. We consider the transmission geometry, when the coupling of the QD to the leads increases to a strong regime [1] and additional transmission channels are activated. The QD is a multilevel system and the transmission through a higher level (close to the Fermi energy) can be treated as an effective bridge channel. Although the electron transport through the QD is governed by the Kondo effect, interference processes are essential and can produce the Fano-shaped resonances. It is also interesting to analyze the Aharonov-Bohm ring with the Kondo impurity. In such a system one can continuously change the interference conditions by varying a magnetic flux and can observe the resulting evolution of the current characteristics from the Kondo peak to the Fano dip. The studies have been performed for various energies of the impurity state: in the Kondo regime, in the mixed-valence regime as well as in the empty state regime.

Our model is described by the Hamiltonian

$$
H=\sum_{k, \sigma, \alpha \in L, R} \epsilon_{k \alpha} c_{k \alpha, \sigma}^{\dagger} c_{k \alpha, \sigma}
$$

$$
\begin{array}{r}
+\sum_{\sigma}\left[\epsilon_{0} c_{0 \sigma}^{\dagger} c_{0 \sigma}+\frac{U}{2} c_{0 \sigma}^{\dagger} c_{0 \sigma} c_{0-\sigma}^{\dagger} c_{0-\sigma}\right] \\
+\sum_{k, \sigma}\left[t_{L 0} c_{k L, \sigma}^{\dagger} c_{0 \sigma}+t_{R 0} c_{k R, \sigma}^{\dagger} c_{0 \sigma}+\text { h.c. }\right] \\
+\sum_{k, k^{\prime}, \sigma}\left[t_{L R} c_{k L, \sigma}^{\dagger} c_{k^{\prime} R, \sigma}+\text { h.c. }\right]
\end{array}
$$

The first term describes electrons in the in the left (L) and the right $(\mathrm{R})$ electrode; the second one describes the quantum dot with a single state $\epsilon_{0}$ and Coulomb interactions characterized by the parameter $U$; the third one corresponds to the tunneling from the electrodes to the dot; and the last one describes the bridge channel over the dot.

The current from the left electrode can be calculated from the time evolution of the occupation number $N_{L}=\sum_{k, \sigma} c_{k L, \sigma}^{\dagger} c_{k L, \sigma}$ for electrons in the left electrode using the Green functions of the Keldysh type [12]. The result is

$$
\begin{array}{r}
J=\frac{2 e}{\hbar} \int \frac{d \omega}{2 \pi} \operatorname{Re}\left[\sum_{k, \sigma} t_{L 0} G_{0, k L \sigma}^{<}(\omega)\right. \\
\left.+\sum_{k, k^{\prime}, \sigma} t_{L R} G_{k^{\prime} R \sigma, k L \sigma}^{<}(\omega)\right]
\end{array}
$$

where $G_{0, k L \sigma}^{<}(\omega)$ and $G_{k^{\prime} R \sigma, k L \sigma}^{<}(\omega)$ are the lesser Green functions corresponding to the states at the dot and in the left electrode or the states in both the electrodes, respectively. Next, we use the Dyson equation to calculate the non-equilibrium Green functions and express $J$ only by the Green function $G_{00}$ at the dot and the bare Green functions $g_{\alpha}$ in the electrodes. The lesser, retarded and advanced Green functions $g_{\alpha}$ are taken in the form $g_{\alpha}^{<}=2 i \pi \rho f_{\alpha}$ and $g_{\alpha}^{r, a}=\mp i \pi \rho$, where $f_{\alpha}$ denotes the Fermi distribution function for electrons in the $\alpha$-electrode and $\rho$ is the density of states. All multiple scatterings on the dot and the contacts, as well as interference processes, are taken into account. Assuming quasi-elastic transport, for which the current conservation rule is fulfilled for any energy $\omega$, one obtains 


$$
\begin{array}{r}
J=\frac{2 e}{h} \int d \omega\left[f_{L}(\omega)-f_{R}(\omega)\right]\left\{\alpha_{L R}\left|t_{L R}\right|^{2}\right. \\
\left.+\operatorname{Im}\left[\alpha_{00}\right] \operatorname{Re}\left[G_{00}^{r}(\omega)\right]+\operatorname{Re}\left[\alpha_{00}\right] \operatorname{Im}\left[G_{00}^{r}(\omega)\right]\right\},
\end{array}
$$

where $\alpha_{L R}=4 \pi^{2} \rho^{2} / w^{2}, \alpha_{00}=-4 \pi \rho z_{L}^{-} z_{L}^{+*} z_{R}^{-} z_{R}^{+*} /$ $\left[w^{2}\left(\left|z_{L}\right|^{2}+\left|z_{R}\right|^{2}\right)\right], w=1+\pi^{2} \rho^{2}\left|t_{L R}\right|^{2}, z_{L}^{ \pm}=t_{L 0} \pm$ $i \pi \rho t_{L R} t_{R 0}^{*}, z_{R}^{ \pm}=t_{R 0}^{*} \pm i \pi \rho t_{L R}^{*} t_{L 0}$. The formula (3) includes two new terms: the first one corresponding to the current through the bridge channel and the second one proportional to $\operatorname{Re} G_{00}^{r}(\omega)$, which is responsible for the Fano resonance [5].

In order to determine the Green function $G_{00}^{r}$ we choose, among a few known approaches 顿, the equation of motion (EOM) method. Although the EOM describes the Kondo resonance only qualitatively, it takes into account all relevant interference processes and can be applied straightforwardly for our model (11) with the bridge channel. The method generates higher-order Green functions, which are truncated according to the self-consistent decoupling procedure proposed by Lacroix [13]. In the limit $U \rightarrow \infty$ the Green function at the dot is determined as

$$
G_{00}^{r}(\omega)=\frac{1-n / 2-a_{00}}{\omega-\epsilon_{0}+i \Delta_{0}-2 i \Delta_{0} a_{00}-b_{00}},
$$

where $\Delta_{0}=\pi \rho\left(\left|t_{L 0}\right|^{2}+\left|t_{R 0}\right|^{2}\right), a_{00}=\left(\left|z_{L}\right|^{2} H_{L}(\omega)+\right.$ $\left.\left|z_{R}\right|^{2} H_{R}(\omega)\right) / w$ and $b_{00}=\left(\left|z_{L}\right|^{2} F_{L}(\omega)+\left|z_{R}\right|^{2} F_{R}(\omega)\right) / w$. Here, we use the functions

$$
\begin{array}{r}
H_{\alpha}(\omega)=\frac{\rho}{w} \int_{-D}^{D} \frac{d \omega^{\prime} f_{\alpha}\left(\omega^{\prime}\right)\left[G_{00}^{r}\left(\omega^{\prime}\right)\right]^{*}}{\omega-\omega^{\prime}}, \\
F_{\alpha}(\omega)=\frac{\rho}{w} \int_{-D}^{D} \frac{d \omega^{\prime} f_{\alpha}\left(\omega^{\prime}\right)}{\omega-\omega^{\prime}}= \\
\frac{\rho}{w}\left\{i \pi f_{\alpha}(\omega)+\ln \frac{2 \pi k_{B} T}{D}+\operatorname{Re} \Psi\left[\frac{1}{2}-i \frac{\omega-\epsilon_{F \alpha}}{2 \pi k_{B} T}\right]\right\},
\end{array}
$$

where $\Psi$ is the digamma function and $\epsilon_{F \alpha}$ denotes the position of the Fermi level in the $\alpha$ electrode. Derivations were performed for the constant density of states $\rho(\epsilon)=1 / 2 D$ for $|\epsilon|<D$ (in our further calculations $D$ is taken as unity). The Green function (位) is similar to that one obtained by Lacroix [13] if one exchanges the tunneling matrix $t_{\alpha 0}$ by an effective one $z_{\alpha}^{r}$. The electron concentration at the dot is given by

$$
n=-\frac{2}{\pi} \int d \omega f_{0}(\omega) \operatorname{Im}\left[G_{00}^{r}(\omega)\right],
$$

where $f_{0}(\omega)=\gamma_{L} f_{L}(\omega)+\gamma_{R} f_{R}(\omega)$ is the non-equilibrium distribution function at the $\operatorname{dot}, \gamma_{L}=\left|z_{L}\right|^{2} /\left(\left|z_{L}\right|^{2}+\right.$ $\left.\left|z_{R}\right|^{2}\right)$ and $\gamma_{R}=\left|z_{R}\right|^{2} /\left(\left|z_{L}\right|^{2}+\left|z_{R}\right|^{2}\right)$.

At $T=0$ the functions $H_{\alpha}(\omega)$ and $F_{\alpha}(\omega)$ have logarithmic singularities at $\omega=\epsilon_{F \alpha}$, but $G_{00}(\omega)$ varies more smoothly around this point. At equilibrium the equation (任) can be written as

$$
G_{00}^{r}\left(\epsilon_{F}\right)=\frac{\left[G_{00}^{r}\left(\epsilon_{F}\right)\right]^{*}}{2 i \Delta_{0}\left[G_{00}^{r}\left(\epsilon_{F}\right)\right]^{*}+1}
$$

A solution of this equation is $G_{00}^{r}\left(\epsilon_{F}\right)=\left[1-e^{2 i \phi}\right] /\left(2 i \Delta_{0}\right)$, where the phase $\phi$ is taken according to the Friedel sum rule [4] as $\phi=\pi n / 2$. On insertion of $G_{00}^{r}$ into (3) one can find the conductance (for the zero bias voltage $V \rightarrow 0$ )

$$
\mathcal{G}=\frac{2 e^{2}}{h}\left\{\alpha_{L R}\left|t_{L R}\right|^{2}-\frac{1}{2 \Delta_{0}} \operatorname{Re}\left[\alpha_{00}\left(1-e^{i \pi n}\right)\right]\right\}
$$

The electron concentration $n$ can be associated with the relative position of the level $\Delta \epsilon=\epsilon_{F}-\epsilon_{0}$ as [4] $n=\frac{1}{2}+\frac{1}{\pi} \tan ^{-1}\left[\Delta \epsilon / \Delta_{0}\right]$, which can be varied by the gate voltage applied to the dot.

For finite temperatures the set of the self-consistent equations (4), (可) and (7) is solved numerically. Figure 1a presents the results for the zero-bias conductance $\mathcal{G}$ through the system with the Kondo dot only $\left(t_{L R}=0\right)$. The Kondo temperature is within the EOM 13. $T_{K}=0.57 \exp \left(-\pi \Delta \epsilon / \Delta_{0}\right) / k_{B} \rho$ and the cross-over from the mixed-valence to the Kondo regime is at $\Delta \epsilon_{c r}=$ $\Delta_{0} / \pi-\Delta_{0} / \pi \ln \left(\rho \Delta_{0} / 2 \pi\right)$, which gives $\Delta \epsilon_{c r} \approx 0.056$ and $\max \left(T_{K}\right) \approx 0.004$ for the parameters used in Fig.1. At $T=0, \mathcal{G}$ is a step-like function, whose maximum value

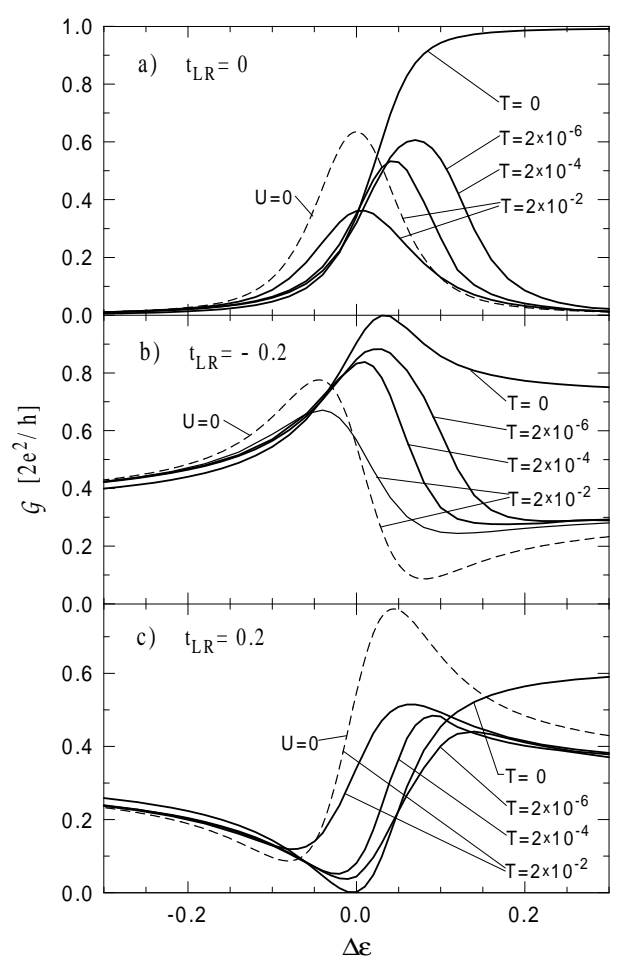

FIG. 1. Conductance through the Kondo system with $t_{L R}=0$ (Fig.a), $t_{L R}=-0.2$ (Fig.b) and $t_{L R}=0.2$ (Fig.c) as a function of $\Delta \epsilon$ for $T=0,2 \times 10^{-6}, 2 \times 10^{-4}$ and $2 \times 10^{-2}$. For comparison $\mathcal{G}$ is shown for the dot without Coulomb interactions $(U=0)$ at a high temperature $T=0.02$ (the dashed curves). In all our calculations $t_{L 0}=0.1, t_{R 0}=0.1$, for which $\Delta_{0}=0.031$. 
$2 e^{2} / h$ is reached in the Kondo regime (see also [14]). At very high temperature $T \gg T_{K}$ Coulomb interactions are irrelevant, and the conductance peak is at $\Delta \epsilon=0$ (see the curve corresponding to $T=0.02$ in Fig.1a). When $T<T_{K}$ the peak is shifted to the Kondo regime and increases logarithmically for $T \rightarrow 0$. Such a shift was observed in many experiments on quantum dots [15]. Since the EOM underestimates temperature dependences, one can expect more pronounced temperature changes for $\mathcal{G}$ than predicted by this method.

Figures $1 \mathrm{~b}$ and $1 \mathrm{c}$ present the results for the system with the bridge channel. The conductance curves have an asymmetric shape, which is typical for a Fano resonance. The effect can be clearly visible for $\left|t_{L R}\right| \gtrsim\left|t_{L 0}\right|,\left|t_{R 0}\right|$. If $t_{L R}$ is negative (Fig.1b), $\mathcal{G}$ exhibits a large maximum, whereas a deep minimum exists for positive values of $t_{L R}$ (Fig.1c). This results from constructive and destructive interference processes for electrons transmitted through two channels. Comparing $\mathcal{G}$ for the interacting and the noninteracting case (the solid and the dashed curves, respectively, in Fig.1) one sees that correlations on the dot weaken the Fano resonance effect (the maximum and the minimum for the solid curves are smaller in the Kondo regime than those for the dashed curves).

In a similar way we calculated the source-drain voltage characteristics of the device. It was assumed that the potential $V$ is applied to the left electrode and in the right electrode the potential is kept zero. Figure 2 presents the evolution of the differential conductance $d I / d V$ with the variation of the relative position of the impurity level $\Delta \epsilon$, from the Kondo to the mixed-valence regime. The case for the pure Kondo dot $\left(t_{L R}=0\right)$ is given in Fig.2a. If the impurity level lies in the Kondo regime, the curves show a very narrow peak at low voltage due to the Anderson hybridization. The sharp feature in the mixedvalence regime (the dash-dotted curve in Fig.2a) we attribute to the EOM method. The peak disappears when $\Delta \epsilon$ approaches the empty state regime (see the dotted curve). Out-equilibrium the Kondo peak is split into two peaks, which are pinned to $\epsilon_{F}+e V$ and $\epsilon_{F}$ (i.e. to the chemical potentials of the left and the right electrode, respectively). This is manifested in the narrow peak of $d I / d V$, which was observed experimentally as well [15]. The broad maximum seen in Fig.2a results from the resonant tunneling when the chemical potential $\epsilon_{F}+e V$ approaches $\epsilon_{0}$.

The influence of the bridge channel is presented in Figs.2b and 2c. A direct electron transmission increases the differential conductance (for our case the bridge channel contribution to $\mathcal{G}$ is $\left.0.33 \times 2 e^{2} / h\right)$. The $d I / d V$ curves in Fig.2b are similar to those in Fig.2a. Although the broad resonance maxima are deformed, there are well pronounced narrow peaks in the low voltage regime. The situation for $t_{L R}=0.2$, presented in Fig.2c, is, however, different. In the low voltage range the curves show a narrow dip instead of a peak. In this case there is a

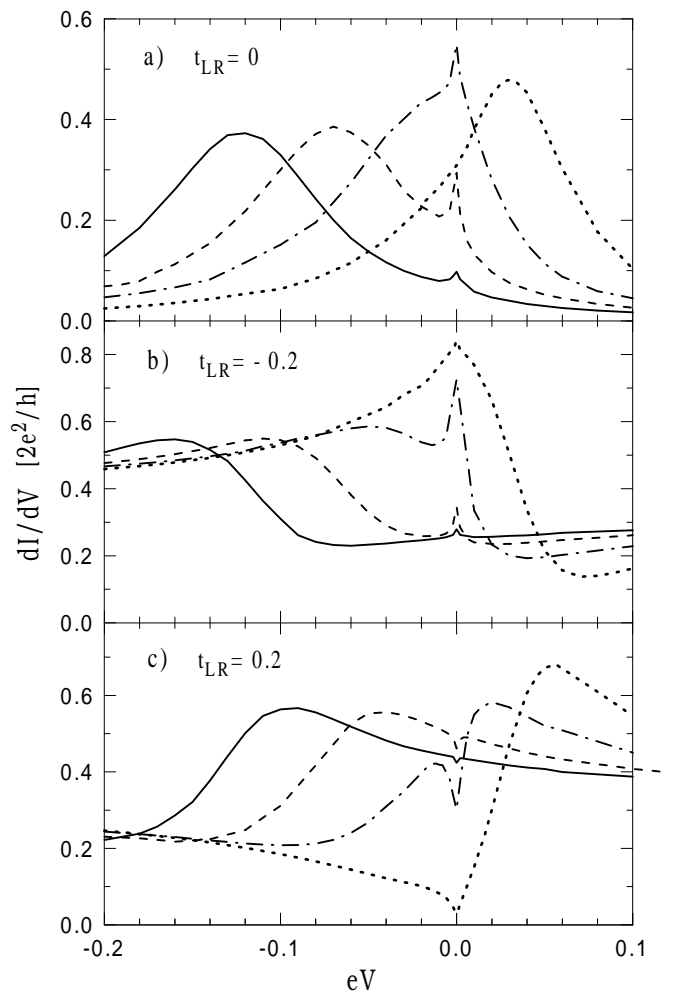

FIG. 2. Differential conductance as a function of the applied voltage for $\Delta \epsilon=0.15$ (solid curve), 0.1 (dashed curve), 0.05 (dash-dotted curve) and 0 (dotted curve) at $T=2 \times 10^{-4}$.

destructive interference of electronic waves passing through the bridge and the Kondo dot. If a small voltage is applied the transmission through the Kondo dot is lowered, which weakens interference of the two channels. It results in the opening of the bridge channel and increase of the conductance. We found that temperature and voltage characteristics for the dip are similar to those for the low voltage peak in the Kondo dot. It is not surprising as the Kondo resonance plays a crucial role in both the situations.

Recent experiments performed by Göres et al. [1] showed that the gray-scale plot of $d I / d V$ in the plane of the gate voltage and the source-drain voltage has a diamond-shaped structure. The behavior is familiar to that one found in the Kondo dot, however, it is a negative picture with dips in place of peaks. As we have explained above, the picture results from weakening of destructive interference processes in the system and opening the bridge channel.

Let us now analyze electron transport though the Aharonov-Bohm ring with the Kondo dot. In this case the hopping integrals are complex numbers $t_{\nu}=\left|t_{\nu}\right| e^{i \phi_{\nu}}$ $(\nu=L R, L 0, R 0)$, where $\phi_{\nu}$ corresponds to the phase of electronic wave passing through the $\nu$ arm of the ring in presence of the magnetic field. At $T=0$ the conductance is given by Eq.(9), which simplifies the Kondo regime $(n=1)$ to the form 


$$
\begin{array}{r}
\mathcal{G}=\frac{2 e^{2}}{h} \frac{4\left|t_{L 0}\right|^{2}\left|t_{R 0}\right|^{2}}{\left(\left|t_{L 0}\right|^{2}+\left|t_{R 0}\right|^{2}\right)^{2} w^{2}}\left[1+\pi^{4} \rho^{4}\left|t_{L R}\right|^{4}\right. \\
\left.-2 \pi^{2} \rho^{2}\left|t_{L R}\right|^{2} \cos \left(2 \pi \Phi / \Phi_{0}\right)\right] .
\end{array}
$$

Here, $\Phi=\left(\phi_{L R}-\phi_{L 0}-\phi_{R 0}\right) / 2 \pi \Phi_{0}$ is the magnetic flux enclosed in the ring and $\Phi_{0}=h c / e$ denotes the one-electron flux quantum. For $T>0$ the electronic transport is calculated numerically and the results are presented in Fig.3. The conductance shows oscillations with $\Phi$ (Fig.3a) with a large amplitude in the mixedvalence regime. In the Kondo regime the amplitude is smaller, but strongly temperature-dependent. Figure 3b presents evolution of the voltage dependence of $d I / d V$ with the magnetic flux $\Phi$. At $\Phi=0$ there is a dip in the curve, which is continuously transformed to a peak for $\Phi=0.5 h c / e$.

Just recently Van der Wiel et al. [9] have performed an experiment on electron transport through the AharonovBohm ring made in two-dimensional electron gas with a quantum dot in one of the arms. The zero-bias conductance, in their experiment, increases considerably and can even reach the value of $2 e^{2} / h$ in some magnetic field ranges. It is in agreement with our results in Fig.3a, where $\mathcal{G}$ can increase to $2 e^{2} / h$ for the Kondo dot if $T \rightarrow 0$ (see also [8]). We expect that this type of experiment [7.9] should also show transformation from a peak to a dip in the voltage dependence of $d I / d V$ as a function of flux through the ring (as seen in Fig.3b).
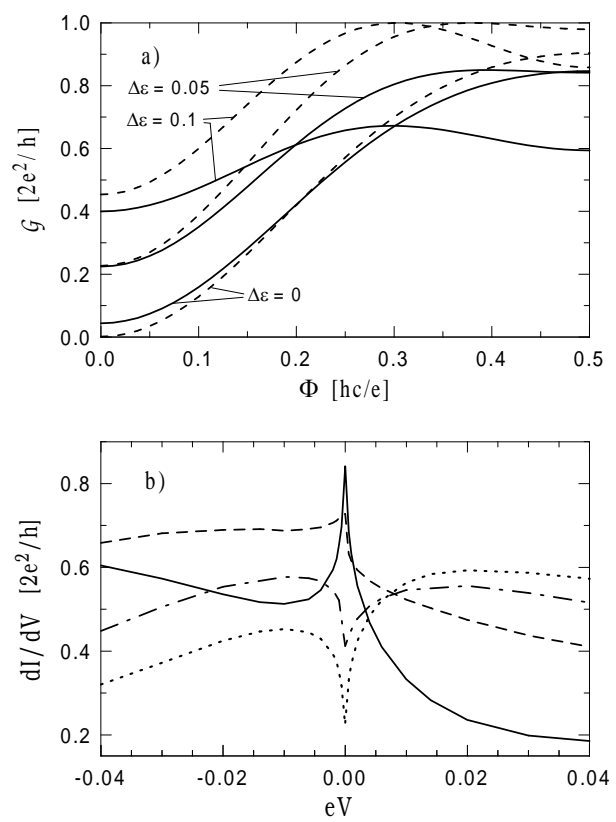

FIG. 3. Characteristics of the Aharonov-Bohm ring with the Kondo dot: Fig.a - the zero-bias conductance vs. the magnetic flux for $\Delta \epsilon=0.1,0.05,0$ at $T=2 \times 10^{-6}$ (solid curves) and $T=0$ (dashed curves); Fig.b - the differential conductance vs. the source-drain voltage for $\Phi=0.5 h c / e$ (solid), 0.25hc/e (dashed) , 0.125hc/e (dash-dotted) and 0 (dotted) at $T=2 \times 10^{-6}, \Delta \epsilon=0.05$.
Summarizing, our theoretical studies of the electronic transport through the quantum dot of the Kondo type showed that interference of travelling waves with the localized state can lead to the Fano effect, for which the current characteristics are strongly modified in the Kondo regime. The source-drain voltage dependence of the conductance exhibits a large peak or a dip, depending on the interference conditions. We predict that interference effects should be pronouncedly seen in transport through the Aharonov-Bohm ring with the Kondo dot.

We have benefitted from discussions with Gerd Czycholl and Tomasz Kostyrko. The work was supported by the Committee for Scientific Research (KBN) under Grant No. 2 P03B 08719.

Note added.- After submission of this Letter we learned of work by Heemeyer [16], in which qualitatively similar results were obtained for the zero-bias conductance (as in Fig.1) within the mean-field slave-boson approach for the model with the bridge channel.

* In the present version a typing error in the coefficient $\alpha_{00}$ [defined after Eq.(3)] has been corrected (the denominator should have $\left.w^{2}\right)$.

[1] J. Göres, et al., Phys. Rev. B 62, 2188 (2000).

[2] W.-D. Schneider, Pramana J. Phys. 52, 537 (1999); J. T. Li, et al., Phys. Rev. Lett. 80, 2893 (1998).

[3] V. Madhavan, et al., Science 280, 567 (1998).

[4] A. C. Hewson, The Kondo problem to heavy fermions (Cambridge University Press, Cambridge 1993).

[5] U. Fano, Phys. Rev. 124, 1866 (1961).

[6] E. Tekman and P. F. Bagwell, Phys. Rev. B 48, 2553 (1993); J. U. Nöckel and A. D. Stone, ibid. B 50, 17415 (1994).

[7] A. Yacoby, et al., Phys. Rev. Lett. 74, 4047 (1995); Y. $\mathrm{Ji}$, et al., cond-mat/0007332v2.

[8] U. Gerland, et al., Phys. Rev. Lett. 84, 3710 (2000).

[9] W. G. van der Wiel, et al., Science 289, 2105 (2000).

[10] A. Schiller and S. Hershfield, Phys. Rev. B 61, 9036 (2000).

[11] O. Újsághy, et al., Phys. Rev. Lett. 85, 2557 (2000).

[12] Y. Meir and N. S. Wingreen, Phys. Rev. Lett. 68, 2512 1992; A.-P. Jauho, et al., Phys. Rev. B 50, 5528 (1994); H. Haug and A.-P. Jauho, Quantum Kinetics in Tranport and Optics of Semiconductors (Springer Verlag, Berlin Heildelberg New York, 1998).

[13] C. Lacroix, J. Phys. F 11, 2389 (1981).

[14] L. I. Glazman and M. E. Raikh, Pis'ma Zh. Eksp. Teor. Fiz. 47, 378 (1988) [JETP Lett. 47, 452 (1988)]; T. K. $\mathrm{Ng}$ and P. A. Lee, Phys. Rev. Lett. 61, 1768 (1988); A. Kawabata, J. Phys. Soc. Jpn. 60, 3222 (1991).

[15] D. Goldhaber-Gordon, et al., Phys. Rev. Lett. 81, 5225 (1998); D. C. Ralph and R. A. Buhrman, ibid. 72, 3401 (1994); S. M. Cronenwett, at al., Science 281, 540 (1998); L. P. Rokhinson, et al., Phys. Rev. B 60, 16319 (1999).

[16] S. Heemeyer, Ph.D. thesis, Massachusetts Institute of Technology 2000. 